\title{
CERTAIN IMBEDDINGS OF WEIGHTED SOBOLEV SPACES
}

\author{
PANKAJ JAIN, BINDU BANSAL AND PAWAN K. JAIN
}

\begin{abstract}
We characterize weight functions for which the weighted Sobolev space $W^{1, p}\left(\Omega, d_{M}^{\beta}\right)$ [and also $W^{1, p}\left(\Omega, s_{0}\left(d_{M}\right)\right)$ ] is imbedded continuously or compactly into the weighted Lebesgue space $L^{q}\left(\Omega, d_{M}^{\alpha}\right)$ [and also $\left.L^{q}\left(\Omega, s_{1}\left(d_{M}\right)\right)\right]$ where $1 \leqslant q<p<\infty$ and $M \subset \partial \Omega$. Some of the imbeddings are also extended to the higher order weighted Sobolev spaces.
\end{abstract}

Mathematics subject classification (2000): 46E35.

Key words and phrases: weighted Sobolev spaces, continuous imbedding, compact imbedding.

\section{REFERENCES}

[1] P. GuRKA AND B. OPIC, Continuous and compact imbeddings of weighted Sobolev spaces I, Czech. Math. J. 38 (113) (1988), 730-744.

[2] P. GuRKA AND B. OpIC, Continuous and compact imbeddings of weighted Sobolev spaces II, Czech. Math. J. 39 (114) (1989), 78-94.

[3] P. JAIn, B. BANSAL AND P. K. JAIN, Continuous and compact imbeddings of weighted Sobolev spaces, Acta Sci. Math. (Szeged) 66 (2000), 665-677.

[4] P. JAIn, B. BANSAL AND P. K. JAIN, Certain imbeddings of Sobolev spaces with power type weights, Indian J. Math., to appear.

[5] A. KuFNER, Weighted Sobolev Spaces, John Wiley \& Sons, Chichester-New York-Brisbane-Toronto-Singapore, 1985.

[6] B. OpIC And A. Kufner, Hardy-Type Inequalities, Pitman Research Notes in Mathematics 219 (1990), Longman, Harlow.

[7] B. OPIC, Necessary and sufficient conditions for imbeddings in weighted Sobolev spaces, Časopis Pěst Mat. 114 (4) (1989), 343-355.

[8] J. RÁKOSNÍK, On imbeddings of Sobolev spaces with power type weights, Theory of Approximation of Functions (1987), 505-507, Nauka, Moscow.

[9] J. RÁKOSNÍK, Weighted Sobolev spaces, preprint. 